\title{
Spectral stability of nonlinear waves in KdV-type evolution equations
}

\author{
Dmitry Pelinovsky \\ Department of Mathematics, McMaster University, Hamilton, Ontario, Canada, L8S 4 K1
}

February 27, 2018

\begin{abstract}
This paper concerns spectral stability of nonlinear waves in KdV-type evolution equations. The relevant eigenvalue problem is defined by the composition of an unbounded self-adjoint operator with a finite number of negative eigenvalues and an unbounded non-invertible operator $\partial_{x}$. The instability index theorem is proven under a generic assumption on the self-adjoint operator both in the case of solitary waves and periodic waves. This result is reviewed in the context of recent results on spectral stability of nonlinear waves in KdV-type evolution equations.
\end{abstract}

Keywords: spectral stability, solitary wave, periodic wave, generalized eigenvalue problem, instability index count

\section{Introduction}

KdV-type evolution equations are defined by the following nonlinear PDE in $(1+1)$ variables:

$$
\frac{\partial u}{\partial t}=\frac{\partial}{\partial x} E^{\prime}(u), \quad u(t) \in \mathcal{X}
$$

where $E: \mathcal{X} \rightarrow \mathbb{R}$ is a $C^{2}$ functional on a subspace $\mathcal{X}$ of Hilbert space $L^{2}$ associated with the inner product $\langle\cdot, \cdot\rangle$ and an induced norm $\|\cdot\|$. A critical point $\phi \in \mathcal{X}$ of the Hamiltonian functional $E$, defined by $E^{\prime}(\phi)=0$, represents a nonlinear wave of the KdV-type evolution equation. Depending on the phase space $\mathcal{X}, \phi$ can be a solitary wave on an infinite line $\mathbb{R}$ or a $(2 L)$-periodic wave on the fundamental period $[-L, L]$. In what follows before the last section, we consider solitary waves on an infinite line defined in a $L^{2}$-based Sobolev space.

The spectral stability of $\phi$ is determined by the spectrum of the non-self-adjoint eigenvalue problem

$$
\partial_{x} E^{\prime \prime}(\phi) v=\lambda v,
$$

where $\mathcal{L}:=E^{\prime \prime}(\phi)$ is a self-adjoint real-valued operator with a dense domain $D(\mathcal{L})$ in $L^{2}(\mathbb{R})$. Since $\mathcal{L}$ is real-valued, for every eigenvalue $\lambda \in \mathbb{C}$ with $\operatorname{Im}(\lambda) \neq 0$, there is an eigenvalue $\bar{\lambda} \in \mathbb{C}$. We also assume the Hamiltonian symmetry, that is, for every eigenvalue $\lambda \in \mathbb{C}$ with $\operatorname{Re}(\lambda) \neq 0$, there is an eigenvalue $-\lambda \in \mathbb{C}$. For instance, if $E^{\prime \prime}(\phi)$ is invariant under the parity transformation, then the Hamiltonian symmetry holds and if $v(x)$ is the eigenvector of the spectral problem (1.2) for $\lambda$, then $v(-x)$ is the eigenvector of the spectral problem (1.2) for $-\lambda$.

The nonlinear wave $\phi$ is spectrally stable if $\sigma\left(\partial_{x} \mathcal{L}\right) \subset i \mathbb{R}$ and it is spectrally unstable if there is $\lambda_{0} \in \sigma\left(\partial_{x} \mathcal{L}\right)$ such that $\operatorname{Re}\left(\lambda_{0}\right)>0$, where $\sigma\left(\partial_{x} \mathcal{L}\right)$ denotes the spectrum of the non-self-adjoint 
eigenvalue problem (1.2). The corresponding eigenvector $v$ for an eigenvalue $\lambda \in \sigma\left(\partial_{x} \mathcal{L}\right)$ belongs to the function space $D\left(\partial_{x} \mathcal{L}\right) \cap \dot{H}^{-1}(\mathbb{R}) \subset L^{2}(\mathbb{R})$, where $\dot{H}^{-1}(\mathbb{R})$ is the space of all distributions with square integrable anti-derivatives. In other words, if $v \in \dot{H}^{-1}(\mathbb{R})$, then $\partial_{x}^{-1} v \in L^{2}(\mathbb{R})$.

We assume that the unbounded self-adjoint operator $\mathcal{L}$ from $D(\mathcal{L}) \subset L^{2}(\mathbb{R})$ to $L^{2}(\mathbb{R})$ is given by the sum of two operators $\mathcal{L}_{0}$ and $K_{\mathcal{L}}$, where $\mathcal{L}_{0}$ is a strongly elliptic unbounded operator with constant coefficients and $K_{\mathcal{L}}$ is a relatively compact perturbation of $\mathcal{L}_{0}$. Using the Fourier transform $\mathcal{F}$ on $L^{2}(\mathbb{R})$, we define the image of $\mathcal{L}_{0}$ as follows:

$$
\mathcal{F}\left(\mathcal{L}_{0} u\right)(k)=\hat{\mathcal{L}}_{0}(k) \mathcal{F}(u)(k), \quad k \in \mathbb{R} .
$$

Since $\mathcal{L}_{0}$ is unbounded, a coercivity condition holds to yield $\hat{\mathcal{L}}_{0}(k) \rightarrow \infty$ as $|k| \rightarrow \infty$. We will further assume the following generic assumptions.

(H1) There is $c_{0}>0$ such that $\hat{\mathcal{L}}_{0}(k) \geq c_{0}$ for all $k \in \mathbb{R}$. By Weyl's theorem, this implies that the essential spectrum of $\mathcal{L}$ (denoted as $\sigma_{e}(\mathcal{L})$ ) is bounded away from zero by a positive number.

(H2) The discrete spectrum of $\mathcal{L}\left(\right.$ denoted as $\left.\sigma_{d}(\mathcal{L})\right)$ includes a finite number $n(\mathcal{L})$ of negative eigenvalues with eigenvectors in $D(\mathcal{L})$.

(H3) $\operatorname{Ker}(\mathcal{L})=\operatorname{span}\left\{f_{0}\right\}$ with $f_{0} \in D(\mathcal{L}) \cap D\left(\partial_{x} \mathcal{L} \partial_{x}\right) \cap \dot{H}^{-1}(\mathbb{R})$, so that $\phi_{0}=\partial_{x}^{-1} f_{0} \in L^{2}(\mathbb{R}) 1$.

(H4) $\left\langle\mathcal{L}^{-1} \phi_{0}, \phi_{0}\right\rangle \neq 0$. The value of $\left\langle\mathcal{L}^{-1} \phi_{0}, \phi_{0}\right\rangle$ is finite because $\left\langle f_{0}, \phi_{0}\right\rangle=\left\langle\partial_{x} \phi_{0}, \phi_{0}\right\rangle=0$.

Under these generic assumptions, we obtain the instability index count, which is analogous to instability index count for NLS-type evolution equations [13, 16, 23, 35]. (see also Chapter 4 in [36]). To formulate the theorem, let us define the following numbers for the eigenvalue problem (1.2) with the account of algebraic multiplicity of eigenvalues:

- $N_{r}$ is the number of real positive eigenvalues $\lambda$.

- $N_{c}$ is the number of complex eigenvalues $\lambda$ in the first open quadrant of $\mathbb{C}$.

- $N_{i}^{-}$is the total negative Krein index 2 associated with the number of imaginary (possibly, embedded) eigenvalues $\lambda$ with $\operatorname{Im}(\lambda)>0$.

Our main result is the following theorem.

Theorem 1 Assume (H1)-(H4). Then,

$$
N_{r}+2 N_{c}+2 N_{i}^{-}=n(\mathcal{L})-n_{0},
$$

where $n_{0}=1$ if $\left\langle\mathcal{L}^{-1} \phi_{0}, \phi_{0}\right\rangle<0$ and $n_{0}=0$ if $\left\langle\mathcal{L}^{-1} \phi_{0}, \phi_{0}\right\rangle>0$.

Section 2 contains historical notes devoted to Theorem 1 and other recent relevant results. The proof of Theorem 1 is developed in Section 3. A generalization of Theorem 1 for a periodic nonlinear wave $\phi$ is given in Section 4. Section 5 discusses further possible developments in the area.

\footnotetext{
${ }^{1}$ The restrictive assumption $f_{0} \in D\left(\partial_{x} \mathcal{L} \partial_{x}\right)$ is needed because $f_{0}$ defines later the projection operator $P$ in the generalized eigenvalue problem (3.7).

${ }^{2}$ The negative Krein index of an invariant subspace $E_{\lambda} \subset L^{2}$ of the spectral stability problem (1.2) associated with an eigenvalue $\lambda \in i \mathbb{R}$ is the number of non-positive eigenvalues of $\left\langle\left.\mathcal{L}\right|_{E_{\lambda}} u, u\right\rangle$. These eigenvalues of $\left\langle\left.\mathcal{L}\right|_{E_{\lambda}} u, u\right\rangle$ can not be zero if $\lambda$ is an isolated eigenvalue but may include zero eigenvalue if $\lambda$ is an embedded eigenvalue.
} 


\section{Historical remarks and examples}

The Hamiltonian functional $E(u)$ conserves in time $t$ in the KdV-type evolution equation (1.1). For many $\mathrm{KdV}$-type evolution equations, there exists typically another conserved $C^{2}$ functional $P(u)$, called the momentum functional. For example, for the general fifth-order KdV equation [9, 15,

$$
u_{t}=a_{1} u_{x}-a_{2} u_{x x x}+a_{3} u_{x x x x x}+3 b_{1} u u_{x}-b_{2}\left(u u_{x x x}+2 u_{x} u_{x x}\right)+6 b_{3} u^{2} u_{x}
$$

where $\left(a_{1}, a_{2}, a_{3}, b_{1}, b_{2}, b_{3}\right)$ are real, the energy functional $E(u)$ is well defined in $H^{2}(\mathbb{R})$,

$$
E(u)=\frac{1}{2} \int_{\mathbb{R}}\left(a_{1} u^{2}+a_{2} u_{x}^{2}+a_{3} u_{x x}^{2}+b_{1} u^{3}+b_{2} u u_{x}^{2}+b_{3} u^{4}\right) d x
$$

whereas the momentum functional is $P(u)=\|u\|^{2}$. Without loss of generality, we assume that the phase speed for linear waves in the fifth-order $\mathrm{KdV}$ equation (2.1) is non-negative. Using the Fourier transform $\mathcal{F}$, we express this assumption as follows:

$$
c_{\text {wave }}(k)=a_{1}+a_{2} k^{2}+a_{3} k^{4} \geq 0, \quad k \in \mathbb{R} .
$$

Assumption (2.3) is needed for assumption (H1) in Theorem 11.

Besides the translational parameter $x_{0}$ in $\phi\left(x-x_{0}\right)$, the nonlinear wave $\phi$ has typically another free parameter $c$ for the constant speed. With the account of speed $c$, the nonlinear wave is a critical point of the extended energy functional $E_{c}(u):=E(u)+c P(u)$. The second variation of $E_{c}$ defines the self-adjoint operator $\mathcal{L}_{c}:=E^{\prime \prime}(\phi)+c P^{\prime \prime}(\phi)$.

General stability-instability results for the critical points of $E_{c}(u)$ were obtained in [4, 19, 39], based on the assumption that the self-adjoint linearized operator $\mathcal{L}_{c}$ has exactly one negative eigenvalue and a simple zero eigenvalue. By a different method involving modulation equations, Lyapunov stability of positive travelling waves $\phi$ was also proved by Weinstein [41. In the consequent two influential papers, Pego and Weinstein developed Evans function analysis of spectral stability [33] and analysis of asymptotic stability in exponentially weighted spaces [34] in the context of a generalized $\mathrm{KdV}$ equation.

These general results correspond to the case $n(\mathcal{L})=1$ in Theorem 1 (see also [1] for a recollection of these and many other results). More recently, questions have been raised on spectral stability of KdV-type nonlinear waves in the cases where $n(\mathcal{L})>1$, which are known for equations of the integrable KdV hierarchy [32, 27]. The result of Theorem 1 was already claimed as early as 2006 in the context of the fifth-order $\mathrm{KdV}$ equation (2.1) [12], although the final version of this paper was published without the example of the fifth-order KdV equation [13. Since that time, a weaker result was obtained by Lin [31] and a nearly identical result was outlined very recently by Kapitula \& Stefanov [25]. Periodic waves of KdV-type nonlinear evolution equations were treated in [2, 3, 7, 17, 21, where results similar to Theorem 1 were obtained. Therefore, it makes fair to restore the original proof of Theorem 1 following the lines of 12 and to show how naturally the instability index count for both solitary and periodic waves can be adopted from a general theory in Pontryagin's space [38. This task is achieved in the present paper with the main goal to show the universality and simplicity of the proof of the instability index counts by using the generalized eigenvalue problem (that is, the linear operator pencil in the terminology of the recent review [29]).

In the context of the general fifth-order KdV equation (2.1), specific studies of Lyapunov stability of travelling solitary waves were reported in [18, 22] with the energy-momentum methods. In particular, since the solitary wave satisfies the fourth-order differential equation,

$$
a_{3} \phi^{\prime \prime \prime \prime}-a_{2} \phi^{\prime \prime}+\left(a_{1}+c\right) \phi+\frac{3}{2} b_{1} \phi^{2}-\frac{1}{2} b_{2}\left(2 \phi \phi^{\prime \prime}+\left(\phi^{\prime}\right)^{2}\right)+2 b_{3} \phi^{3}=0,
$$


one can verify by direct computations that $\mathcal{L}_{c} \phi^{\prime}=0$ and $\mathcal{L}_{c} \partial_{c} \phi=-\phi$, where the prime denotes differentiation in $x$ and $\partial_{c}$ denotes differentiation in $c$, whereas

$$
\mathcal{L}_{c}:=a_{3} \frac{d^{4}}{d x^{4}}-a_{2} \frac{d^{2}}{d x^{2}}+a_{1}+c+3 b_{1} \phi(x)-b_{2} \frac{d}{d x} \phi(x) \frac{d}{d x}-b_{2} \phi^{\prime \prime}(x)+6 b_{3} \phi^{2}(x) .
$$

Assuming existence and uniqueness (up to translational invariance) of a solitary wave $\phi \in H^{2}(\mathbb{R})$ with the exponential decay at infinity for $c>0$ (see [9, 26, 30] for existence results), we realize that the operator $\mathcal{L}_{c}$ satisfies assumptions $(\mathrm{H} 1)-(\mathrm{H} 4)$ of Theorem 1 with $\hat{\mathcal{L}}_{0}(k)=c+c_{\text {wave }}(k) \geq c>0$, $\phi_{0}=\phi$, and

$$
\left\langle\mathcal{L}_{c}^{-1} \phi, \phi\right\rangle=-\left\langle\partial_{c} \phi, \phi\right\rangle=-\frac{1}{2} \frac{d}{d c}\|\phi\|^{2} .
$$

If $n\left(\mathcal{L}_{c}\right)=1$, the result of Theorem 1 gives stability of a solitary wave if $\frac{d}{d c}\|\phi\|^{2}>0$ and instability if $\frac{d}{d c}\|\phi\|^{2}<0$, which coincides with the results of the orbital stability theory [1, 4, 41].

Spectral stability of one-humped solitary waves in the fifth-order KdV equation was studied numerically in [6], with the use of the symplectic Evans matrix [5]. Because $n\left(\mathcal{L}_{c}\right)=1$ and $\frac{d}{d c}\|\phi\|^{2}>0$ were found, the one-humped solitary waves were shown to be spectrally stable.

One-humped and two-humped solitary waves in the fifth-order KdV equation were numerically approximated in [11] with a spectral method. Numerical results on eigenvalues of the spectral problem (1.2) were found in full correspondence with the result of Theorem 1, In particular, twohumped solutions have either $n\left(\mathcal{L}_{c}\right)=2$ or $n\left(\mathcal{L}_{c}\right)=3$, depending whether the individual solitary waves form a bound state at the non-degenerate minimum or maximum points of the effective interaction potential. Since $\frac{d}{d c}\|\phi\|^{2}>0$ for all these solitary waves, the two-humped solutions with $n\left(\mathcal{L}_{c}\right)=2$ are unstable with $N_{r}=1$. Nevertheless, the two-humped solutions with $n\left(\mathcal{L}_{c}\right)=3$ are spectrally stable, because the single pair of embedded eigenvalues with negative Krein signature $N_{i}^{-}=1$ is structurally stable with respect to parameter continuations [11]. Similar results were also observed numerically with the computations of the Maslov index for solitary waves in the fifth-order $\mathrm{KdV}$ equation [10].

To finish these remarks, we also mention a similar instability index count obtained for dark solitons in the defocusing NLS equation with an external potential [37]. Although the symplectic operator for the NLS equation is invertible, the spectral stability problem for dark solitons (solitary waves with nonzero boundary conditions) is defined in terms of a linear self-adjoint operator, where the positive essential spectrum touches zero. Nevertheless, the theory from [13] was successfully applied to the count of unstable eigenvalues for a dark soliton in a spatially localized potential and illustrated with a number of prototypical examples in [37]. In this context, a dark soliton persists in a small localized potential if it is located at the non-degenerate minimum or maximum points of the effective potential and is spectrally unstable in both cases. At the maximum point, the dark soliton is unstable with one real eigenvalue $N_{r}=1$, whereas at the minimum point, it is unstable with two complex eigenvalues $N_{c}=1$. The embedded imaginary eigenvalues with negative Krein signature are structurally unstable with respect to parameter continuations in the defocusing NLS equations and bifurcate into complex unstable eigenvalues (see [37] for precise computations of these instabilities by using the Evans function for dark solitons).

\section{Proof of Theorem 1}

We consider the spectral problem (1.2), where the self-adjoint operator $\mathcal{L}=E^{\prime \prime}(\phi)$ satisfies the assumptions (H1)-(H4) of Theorem 1 . 
The proof of the standard instability index count [13, 16, 23, 35] is not applicable to the KdVtype evolution equations because the symplectic operator $\partial_{x}$ is not invertible. Nevertheless, the range of the self-adjoint operator $\mathcal{L}$ is defined in $L^{2}(\mathbb{R})$, hence bootstrapping arguments imply that the eigenvector $v$ of the spectral problem (1.2) with $\lambda \neq 0$ belongs to the function space $D\left(\partial_{x} \mathcal{L}\right) \cap \dot{H}^{-1}(\mathbb{R}) \subset L^{2}(\mathbb{R})$. Therefore, we can define $w=\partial_{x}^{-1} v \in L^{2}(\mathbb{R})$ and formally extend the spectral problem (1.2) to the system of two coupled equations:

$$
\mathcal{M} w=-\lambda v, \quad \mathcal{L} v=\lambda w
$$

where $\mathcal{M}:=-\partial_{x} \mathcal{L} \partial_{x}, v \in D\left(\partial_{x} \mathcal{L}\right) \cap \dot{H}^{-1}(\mathbb{R}) \subset L^{2}(\mathbb{R})$, and $w \in D\left(\partial_{x} \mathcal{L} \partial_{x}\right) \subset L^{2}(\mathbb{R})$. The coupled system (3.1) is equivalent to the squared eigenvalue problem $\partial_{x} \mathcal{L} \partial_{x} \mathcal{L} v=\lambda^{2} v$. We show now that if the coupled system (3.1) has an eigenvalue $\lambda_{0} \neq 0$, then it has another eigenvalue $-\lambda_{0}$ and these two eigenvalues are equivalent to the pair of eigenvalues $\lambda_{0}$ and $-\lambda_{0}$ of the spectral problem (1.2). For simplicity of presentation, we only consider the case of simple nonzero eigenvalues in this work.

Proposition 1 The coupled system (3.1) has a pair of simple eigenvalues $\pm \lambda_{0} \neq 0$ with the eigenvectors $\left(v_{0}, \pm w_{0}\right) \in D\left(\partial_{x} \mathcal{L}\right) \cap \dot{H}^{-1}(\mathbb{R}) \times D\left(\partial_{x} \mathcal{L} \partial_{x}\right)$ if and only if the spectral problem (1.2) has a pair of simple eigenvalues $\pm \lambda_{0}$ with the eigenvectors $v_{ \pm}=v_{0} \pm \partial_{x} w_{0} \in D\left(\partial_{x} \mathcal{L}\right) \cap \dot{H}^{-1}(\mathbb{R})$.

Proof. By the symmetry, if $\lambda_{0} \neq 0$ is a simple eigenvalue of the coupled system (3.1) with the eigenvector $\left(v_{0}, w_{0}\right) \in D\left(\partial_{x} \mathcal{L}\right) \cap \dot{H}^{-1}(\mathbb{R}) \times D\left(\partial_{x} \mathcal{L} \partial_{x}\right)$, then $-\lambda_{0}$ is also a simple eigenvalue of the coupled system (3.1) with the eigenvector $\left(v_{0},-w_{0}\right)$. Moreover, $v_{0}$ and $w_{0}$ are linearly independent.

We differentiate the second equation of the coupled system (3.1) for the eigenvalue $\lambda_{0}$ and add or subtract the first equation of the system to obtain

$$
\partial_{x} \mathcal{L}\left(v_{0} \pm \partial_{x} w_{0}\right)= \pm \lambda_{0}\left(v_{0} \pm \partial_{x} w_{0}\right) .
$$

Therefore, $v_{0} \pm \partial_{x} w_{0} \in \operatorname{Ker}\left(\partial_{x} \mathcal{L} \mp \lambda_{0}\right)$. By the Hamiltonian symmetry, if $\lambda_{0} \in \sigma\left(\partial_{x} \mathcal{L}\right)$, then $-\lambda_{0} \in \sigma\left(\partial_{x} \mathcal{L}\right)$, whereas the algebraic multiplicity of eigenvalues in $\sigma\left(\partial_{x} \mathcal{L} \partial_{x} \mathcal{L}\right)$ equals the algebraic multiplicity of eigenvalues in the coupled system (3.1). This guarantees that the two eigenvectors $\left(v_{0}, \pm w_{0}\right)$ of the coupled system (3.1) generate two linearly independent eigenvectors $v_{ \pm}=v_{0} \pm \partial_{x} w_{0}$ for $\pm \lambda_{0} \in \sigma\left(\partial_{x} \mathcal{L}\right)$.

To check the converse statement, we assume that $v_{ \pm}$are linearly independent eigenvectors of $\partial_{x} \mathcal{L}$ for the eigenvalues $\pm \lambda_{0}$ in $D\left(\partial_{x} \mathcal{L}\right) \cap \dot{H}^{-1}(\mathbb{R})$. Then, we define nonzero functions

$$
v_{0}:=\frac{1}{2}\left(v_{+}+v_{-}\right), \quad w_{0}:=\frac{1}{2}\left(\partial_{x}^{-1} v_{+}-\partial_{x}^{-1} v_{-}\right),
$$

and obtain

$$
\partial_{x} \mathcal{L} v_{0}=\frac{1}{2} \partial_{x} \mathcal{L}\left(v_{+}+v_{-}\right)=\frac{1}{2} \lambda_{0}\left(v_{+}-v_{-}\right)=\lambda_{0} \partial_{x} w_{0},
$$

so that the integration gives $\mathcal{L} v_{0}=\lambda_{0} w_{0}$, that is, the second equation of the coupled system (3.1). Similarly, we check the first equation of the coupled system (3.1) with $\mathcal{M} w_{0}=-\lambda_{0} v_{0}$. Therefore, the two eigenvectors $v_{ \pm}$for $\pm \lambda_{0} \in \sigma\left(\partial_{x} \mathcal{L}\right)$ generate two linearly independent eigenvectors $\left(v_{0}, \pm w_{0}\right)$ of the coupled system (3.1) for a pair of eigenvalues $\pm \lambda_{0} \neq 0$.

Remark 1 In many KdV-type evolution equations including the fifth-order $K d V$ equation (2.1), the Hamiltonian symmetry of the spectral problem (1.2) follows from the parity transformation of the eigenvectors, if the nonlinear wave $\phi$ is symmetric with respect to $x$. Therefore, if $v_{+}(x)$ is a solution of $\partial_{x} \mathcal{L} v_{+}=\lambda_{0} v_{+}$, then $v_{-}(x):=v_{+}(-x)$ is a solution of $\partial_{x} \mathcal{L} v_{-}=-\lambda_{0} v_{-}$. Under this transformation, the components $v_{0}$ and $w_{0}$ of the coupled system (3.1) for a simple eigenvalue $\lambda_{0}$ are either even or odd functions with respect to $x$, whereas $v_{ \pm}$are neither even nor odd. 
To study the spectrum of the coupled system (3.1), we shall first understand the spectrum of operator $\mathcal{M}$. Recall again that $\sigma_{e}$ and $\sigma_{d}$ denote the essential and discrete spectra. From assumptions (H1)-(H4), we obtain the following properties of operator $\mathcal{M}$.

Lemma 2 Under assumptions $\left(H_{1}\right)-\left(H_{4}\right)$ on $\mathcal{L}$, operator $\mathcal{M}$ can be extended to a self-adjoint operator with a dense domain $D(\mathcal{M})$ in $L^{2}(\mathbb{R})$ satisfying the following properties:

$\left(\mathrm{H} 1^{\prime}\right) \sigma_{e}(\mathcal{M}) \geq 0$.

$\left(\mathrm{H} 2^{\prime}\right) \sigma_{d}(\mathcal{M})$ includes $n(\mathcal{L})$ negative eigenvalues with eigenvectors in $D(\mathcal{M})$.

$\left(\mathrm{H} 3^{\prime}\right) \operatorname{Ker}(\mathcal{M})=\operatorname{span}\left\{\phi_{0}\right\}$.

$\left(\mathrm{H} 4^{\prime}\right)\left\langle\mathcal{M}^{-1} f_{0}, f_{0}\right\rangle$ is finite and nonzero.

Proof. From the decomposition $\mathcal{L}=\mathcal{L}_{0}+K_{\mathcal{L}}$, we have the decomposition $\mathcal{M}=\mathcal{M}_{0}+K_{\mathcal{M}}$, where $K_{\mathcal{M}}=-\partial_{x} K_{\mathcal{L}} \partial_{x}$ is a relatively compact perturbation of $\mathcal{M}_{0}=-\partial_{x} \mathcal{L}_{0} \partial_{x}$. Since $\mathcal{M}_{0}$ is a linear operator with constant coefficients, we use the Fourier transform $\mathcal{F}$ on $L^{2}(\mathbb{R})$ to find the image of $\mathcal{M}_{0}$ as follows:

$$
\hat{\mathcal{M}}_{0}(k)=k^{2} \hat{\mathcal{L}}_{0}(k) \geq 0 \quad \text { for all } k \in \mathbb{R} .
$$

Since $\hat{\mathcal{L}}_{0}(k) \geq c_{0}$ by assumption $(\mathrm{H} 1)$, we have $\hat{\mathcal{M}}_{0}(k) \geq 0$ for all $k \in \mathbb{R}$. By Weyl's theorem, this implies that $\sigma_{e}(\mathcal{M})$ is non-negative, that is, $\left(\mathrm{H} 1^{\prime}\right)$ holds.

Since $\operatorname{Ker}\left(\partial_{x}\right)=\operatorname{span}\{0\}$ in $L^{2}(\mathbb{R}),\left(H 3^{\prime}\right)$ follows from (H3) by direct computations:

$$
-\partial_{x} \mathcal{L} \partial_{x} f=0 \Rightarrow \mathcal{L} \partial_{x} f=0 \Rightarrow \partial_{x} f \in \operatorname{span}\left\{\partial_{x} \phi_{0}\right\}, \Rightarrow f \in \operatorname{span}\left\{\phi_{0}\right\}
$$

Furthermore, $\left\langle\mathcal{M}^{-1} f_{0}, f_{0}\right\rangle$ exists because $\partial_{x}^{-1}$ is well defined in $L^{2}(\mathbb{R})$ on functions in $L^{2}(\mathbb{R}) \cap$ $\dot{H}^{-1}(\mathbb{R})$. As a result, $\left(\mathrm{H}^{\prime}\right)$ follows from $(\mathrm{H} 4)$ by means of integration by parts:

$$
\left\langle\mathcal{M}^{-1} f_{0}, f_{0}\right\rangle=\left\langle\mathcal{M}^{-1} \partial_{x} \phi_{0}, \partial_{x} \phi_{0}\right\rangle=-\left\langle\partial_{x} \mathcal{M}^{-1} \partial_{x} \phi_{0}, \phi_{0}\right\rangle=\left\langle\mathcal{L}^{-1} \phi_{0}, \phi_{0}\right\rangle .
$$

It remains to prove $\left(\mathrm{H} 2^{\prime}\right)$. The negative eigenvalues of $\sigma_{d}(\mathcal{M})$ are defined from the eigenvalue problem $\mathcal{M} f=\lambda f$, which is rewritten in the following form:

$$
-\partial_{x} \mathcal{L} \partial_{x} f=\lambda f, \quad f \in D\left(\partial_{x} \mathcal{L} \partial_{x}\right) \cap \dot{H}^{-1}(\mathbb{R}) \subset L^{2}(\mathbb{R}) .
$$

Since $f \in \dot{H}^{-1}(\mathbb{R})$, there exists $g=\partial_{x}^{-1} f \in L^{2}(\mathbb{R})$ such that the spectral problem (3.3) can be written in the equivalent form

$$
-\mathcal{L} \partial_{x}^{2} g=\lambda g, \quad g \in D\left(\mathcal{L} \partial_{x}^{2}\right) \subset L^{2}(\mathbb{R}) .
$$

For any $\epsilon>0$, the positive operator $\left(\epsilon-\partial_{x}^{2}\right)$ is invertible and the inverse operator is defined by the integral representation

$$
\left(\epsilon-\partial_{x}^{2}\right)^{-1} f(x)=\frac{1}{2 \epsilon^{1 / 2}} \int_{\mathbb{R}} e^{-\epsilon^{1 / 2}|x-y|} f(y) d y, \quad f \in L^{2}(\mathbb{R}) .
$$

Using this integral representation, we define a smoothen version of the eigenvalue problem (3.4) for $h=\left(\epsilon-\partial_{x}^{2}\right) g$ :

$$
\mathcal{L} h=\lambda\left(\epsilon-\partial_{x}^{2}\right)^{-1} h, \quad h \in D(\mathcal{L}) \subset L^{2}(\mathbb{R}) .
$$


Since $\left(\epsilon-\partial_{x}^{2}\right)^{-1}$ is a positive bounded self-adjoint operator for any $\epsilon>0$, Sylvester's Law of Inertia (Theorem 4.2 in [36]) applies and the number of negative and zero eigenvalues of the spectral problem (3.6) corresponds to the number of negative and zero eigenvalues of the operator $\mathcal{L}$. By assumptions $(\mathrm{H} 2)$ and $(\mathrm{H} 3)$, there are exactly $n(\mathcal{L})$ negative eigenvalues of the eigenvalue problem (3.6) and a simple zero eigenvalue for any $\epsilon>0$.

Because the integral representation (3.5) diverges as $\epsilon \downarrow 0$ and the operator $\mathcal{L}$ is bounded from below, the negative eigenvalues of the spectral problem (3.6) for $\epsilon>0$ are bounded from below but may a priori approach to zero as $\epsilon \downarrow 0$. However, since the kernel of $\left(\epsilon-\partial_{x}^{2}\right) \mathcal{L}$ is simple for any $\epsilon \geq 0$, the negative eigenvalues are bounded away from zero as $\epsilon \downarrow 0$. As a result, the spectral problem (3.4) also has $n(\mathcal{L})$ negative eigenvalues, that is, $\left(\mathrm{H}^{\prime}\right)$ hold 3 .

We convert now the coupled system (3.1) for a pair of simple eigenvalues $\pm \lambda \neq 0$ to a generalized eigenvalue problem for a double eigenvalue. By assumptions (H1) and (H3), the zero eigenvalue of $\mathcal{L}$ is bounded away from the essential spectrum of $\mathcal{L}$. Let $P$ be the orthogonal projection from $L^{2}(\mathbb{R})$ to $\left[\operatorname{span}\left\{f_{0}\right\}\right]^{\perp} \subset L^{2}(\mathbb{R})$. The following result establishes this equivalence.

Proposition 3 The coupled system (3.1) has a pair of simple eigenvalues $\pm \lambda \neq 0$ with eigenvectors $(v, \pm w)$ if and only if the generalized eigenvalue problem

$$
A w=\gamma K w, \quad w \in \mathcal{H}:=D(\mathcal{M}) \cap\left[\operatorname{span}\left\{f_{0}\right\}\right]^{\perp} \subset L^{2}(\mathbb{R}),
$$

where $A:=P \mathcal{M P}$ and $K:=P \mathcal{L}^{-1} P$, has a double eigenvalue $\gamma=-\lambda^{2} \neq 0$ with linearly independent eigenvectors $\partial_{x}^{-1} v$ and $w$.

Proof. Let $\pm \lambda \neq 0$ be a pair of simple eigenvalues of the coupled system (3.1) with the eigenvectors $(v, \pm w) \in D\left(\partial_{x} \mathcal{L}\right) \cap \dot{H}^{-1}(\mathbb{R}) \times D\left(\partial_{x} \mathcal{L} \partial_{x}\right)$. Because $\lambda \neq 0$, we have $w=P w$, that is, $w$ is in the range of $\mathcal{L}$. As a result, the second equation of the coupled system (3.1) can be written in the equivalent form:

$$
v=\lambda P \mathcal{L}^{-1} P w+v_{0}, \quad v_{0} \in \operatorname{Ker}(\mathcal{L}) .
$$

Substituting $v$ into the first equation of the coupled system (3.1) and using the projection operator $P$ again, we obtain a closed equation for $w$

$$
P \mathcal{M} P w=-\lambda^{2} P \mathcal{L}^{-1} P w, \quad w \in D(\mathcal{M}) \cap\left[\operatorname{span}\left\{f_{0}\right\}\right]^{\perp} \subset L^{2}(\mathbb{R})
$$

and a unique expression for $v_{0}$

$$
v_{0}=-\frac{1}{\lambda}(I-P) \mathcal{M P w}
$$

where $\lambda \neq 0$ and $(I-P)$ is the orthogonal projection from $L^{2}(\mathbb{R})$ to $\operatorname{Ker}(\mathcal{L})$. Therefore, it follows from equation (3.9) that $\gamma=-\lambda^{2}$ is an eigenvalue of the generalized eigenvalue problem (3.7) with an eigenvector $w$.

To show that this $\gamma$ is a double eigenvalue of the generalized eigenvalue problem (3.7), we note that the coupled system (3.1) is invariant with respect to the transformation

$$
\partial_{x} w \rightarrow v \quad \text { and } \quad \partial_{x}^{-1} v \rightarrow w .
$$

\footnotetext{
${ }^{3}$ Another smoothen version of the same eigenvalue problem (3.4) is $\left(\epsilon-\partial_{x}^{2}\right) g=\lambda \mathcal{L}^{-1} g$ for all $g \in H^{2}(\mathbb{R}) \cap$ $\left[\operatorname{span}\left\{f_{0}\right\}\right]^{\perp}$. By the same Sylvester's Law of Inertia, there are exactly $n(\mathcal{L})$ negative eigenvalues of this eigenvalue problem for any $\epsilon>0$ and the bootstrapping arguments give $g \in D\left(\mathcal{L} \partial_{x}^{2}\right) \subset L^{2}(\mathbb{R})$ for the corresponding eigenvectors.
} 
Therefore, $\partial_{x}^{-1} v$ is another eigenvector of the generalized eigenvalue problem (3.7) for the same $\gamma$. By Proposition 1, see the equivalence formula (3.2), $\partial_{x}^{-1} v$ and $w$ are linearly independent.

In the opposite direction, if $\gamma \neq 0$ is a double eigenvalue of the generalized eigenvalue problem (3.7) with linearly independent eigenvectors $w_{1}$ and $w_{2}$, then for each eigenvector $w_{1}$ or $w_{2}$, we define $v_{0}$ by (3.10) and $v$ by (3.8), which yields linearly independent components $v_{1}$ and $v_{2}$ of the coupled system (3.1) for the eigenvalue $\lambda=(-\gamma)^{1 / 2}$. The eigenvalue $\lambda=(-\gamma)^{1 / 2}$ must be simple (or the multiplicity of the eigenvalue $\gamma$ in the generalized eigenvalue problem (3.7) exceeds two), therefore, the transformation (3.11) yields the correspondence $v_{1}=\partial_{x} w_{2}$ and $v_{2}=\partial_{x} w_{1}$. In other words, only $\left(v_{1}, w_{1}\right)$ is a linearly independent eigenvector of the coupled system (3.1) for the simple eigenvalue $\lambda=(-\gamma)^{1 / 2}$. The other simple eigenvalue $-\lambda=-(-\gamma)^{1 / 2}$ exists by the symmetry of the coupled system (3.1) with the eigenvector $\left(v_{1},-w_{1}\right)$.

The generalized eigenvalue problem (3.7) for unbounded self-adjoint differential operators $A$ and $K$ with strictly positive essential spectrum was studied by Chugunova \& Pelinovsky 13 in Pontryagin's space [38]. Here we report the modification of the analysis needed to treat the case when the bottom of the essential spectrum of $A$ touches zero. We shall first prove that a deformation of $A$ to $A_{\delta}:=A+\delta K$ for a small positive number $\delta$ shifts the essential spectrum away from zero.

Lemma 4 For small positive values of $\delta$, there is a positive $\delta$-independent constant $d_{0}$ such that

$$
\sigma_{e}\left(A_{\delta}\right) \geq d_{0} \delta
$$

Proof. Since $\mathcal{M}$ and $\mathcal{L}$ are represented by the relatively compact perturbations of operators $\mathcal{M}_{0}$ and $\mathcal{L}_{0}$ with constant coefficients, we can use the Fourier transform $\mathcal{F}$ on $L^{2}(\mathbb{R})$ to compute:

$$
\mathcal{F}\left(\mathcal{M}_{0}+\delta \mathcal{L}_{0}^{-1}\right)=k^{2} \hat{\mathcal{L}}_{0}(k)+\delta \hat{\mathcal{L}}_{0}^{-1}(k), \quad k \in \mathbb{R},
$$

where $\hat{\mathcal{L}}_{0}(k) \geq c_{0}>0$ for some $c_{0}$ by $(\mathrm{H} 1)$.

Let $k_{\delta}$ denote the positive global minimum of this function. By coercivity of $k^{2} \hat{\mathcal{L}}_{0}(k)$, the global minimum is achieved at a finite value of $k$ for small positive values of $\delta$ and there is a $\delta$-independent positive constant $K_{0}$ such that $k_{\delta} \in\left[0, K_{0}\right]$. But then, there is a $\delta$-independent positive constant $d_{0}$ such that $\hat{\mathcal{L}}_{0}^{-1}\left(k_{\delta}\right) \geq d_{0}$ and $k^{2} \hat{\mathcal{L}}_{0}(k)+\delta \hat{\mathcal{L}}_{0}^{-1}(k) \geq d_{0} \delta$ for small positive $\delta$.

By Lemma 4, the essential spectrum of $A_{\delta}$ for a small positive $\delta$ is strictly positive. Also, the kernel of $A_{\delta}$ is empty for a small positive $\delta$ because if $f \in \operatorname{Ker}\left(A_{\delta}\right)$, then $A f=-\delta K f$ but the negative eigenvalues do not accumulate near zero, thanks to the decomposition $\mathcal{L}=\mathcal{L}_{0}+K_{\mathcal{L}}$ with a relatively compact perturbation $K_{\mathcal{L}}$. Therefore, there exists a small positive number $\delta$ such that operator $A_{\delta}$ is continuously invertible in $\mathcal{H}$ and the generalized eigenvalue problem (3.7) is rewritten in the shifted form,

$$
(A+\delta K) w=(\gamma+\delta) K w, \quad w \in \mathcal{H} .
$$

By the spectral theory of self-adjoint operators, the Hilbert space $\mathcal{H}$ can be equivalently decomposed into two orthogonal sums of subspaces which are invariant with respect to the operators $K$ and $A_{\delta}$ for small positive values of $\delta$ :

$$
\mathcal{H}=\mathcal{H}_{K}^{-} \oplus \mathcal{H}_{K}^{+}=\mathcal{H}_{A_{\delta}}^{-} \oplus \mathcal{H}_{A_{\delta}}^{+},
$$


where notation $-(+)$ stands for invariant subspaces of these operators related to the negative (positive) spectrum.

Since $P$ is a projection defined by the eigenspace of $\mathcal{L}$ and $K=P \mathcal{L}^{-1} P$, it is obvious that

$$
\operatorname{dim}\left(\mathcal{H}_{K}^{-}\right)=n(\mathcal{L})
$$

On the other hand, the number of negative eigenvalues of $A=P \mathcal{M} P$ is related to the number of negative eigenvalues of $\mathcal{M}$. Compared to the standard count of negative eigenvalues in constrained Hilbert spaces (Theorem 4.1 in [36]), the complication here is that the zero eigenvalue of $\mathcal{M}$ is embedded to the edge of the essential spectrum of $\mathcal{M}$. In addition, the zero eigenvalue of $A$ is shifted under the perturbation $\delta K$ in the operator $A_{\delta}=A+\delta K$. The following two lemmas give the count of negative eigenvalues of $A$ denoted as $n(A)$ and the count of $\operatorname{dim}\left(\mathcal{H}_{A_{\delta}}^{-}\right)$for a small positive number $\delta$.

Lemma 5 Under assumptions $(\mathrm{H} 1)-\left(\mathrm{H}_{4}\right)$ on $\mathcal{L}$, we have

$$
n(A)=n(P \mathcal{M} P)=n(\mathcal{M})-n_{0}=n(\mathcal{L})-n_{0},
$$

where $n_{0}=1$ if $\left\langle\mathcal{L}^{-1} \phi_{0}, \phi_{0}\right\rangle<0$ and $n_{0}=0$ if $\left\langle\mathcal{L}^{-1} \phi_{0}, \phi_{0}\right\rangle>04$.

Proof. We study the behavior of the function $F(\mu)=\left\langle(\mu-\mathcal{M})^{-1} f_{0}, f_{0}\right\rangle$, which is well-defined for all $\mu \in \mathbb{R}_{-} \backslash \sigma(\mathcal{M})$. By $\left(\mathrm{H} 4^{\prime}\right)$, it has the limit as $\mu$ approaches zero from below:

$$
\lim _{\mu \uparrow 0} F(\mu)=-\left\langle\mathcal{M}^{-1} f_{0}, f_{0}\right\rangle=-\left\langle\mathcal{L}^{-1} \phi_{0}, \phi_{0}\right\rangle \neq 0 .
$$

Hence, the assertion of the lemma holds by the standard proof of Theorem 4.1 in [36] (where it is formulated and proved in a more general setting).

Lemma 6 Under assumptions (H1)-(H4) on $\mathcal{L}$, for a small positive number $\delta$, we have

$$
\operatorname{dim}\left(\mathcal{H}_{A_{\delta}}^{-}\right)=n(\mathcal{L})
$$

Proof. Negative eigenvalues of $\sigma_{d}\left(A_{\delta}\right)$ are defined from the eigenvalue problem:

$$
A f+\delta K f=\lambda f \quad f \in \mathcal{H} .
$$

We use the assumptions that $\operatorname{Ker}(A)=\operatorname{span}\left\{\phi_{0}\right\}$ and $\left\langle K \phi_{0}, \phi_{0}\right\rangle=\left\langle\mathcal{L}^{-1} \phi_{0}, \phi_{0}\right\rangle \neq 0$. Since negative eigenvalues of $A$ in Lemma 5 are bounded away from zero, they persist for small positive values of $\delta$. Let $\mathcal{H}_{\delta}$ denote the orthogonal complement of the subspace spanned by $n(A)$ eigenvectors corresponding to these negative eigenvalues of $A+\delta K$ for small positive values of $\delta$.

At $\delta=0$, we have $\phi_{0} \in \mathcal{H}_{\delta=0}$. If $\left\langle K \phi_{0}, \phi_{0}\right\rangle<0$, then $A_{\delta}=A+\delta K$ is not positive definite on $\mathcal{H}_{\delta}$ for small positive $\delta$. Therefore, there is at least one negative (isolated) eigenvalue of $A_{\delta}$, which becomes the zero eigenvalue of $A$ as $\delta \rightarrow 0$ (the zero eigenvalue of $A$ is embedded at the edge of

\footnotetext{
${ }^{4}$ By the standard technique (Theorem 4.1 in 36 ), we also have $n(\tilde{P} \mathcal{L} \tilde{P})=n(\mathcal{L})-n_{0}$, where $\tilde{P}$ is an orthogonal projection from $L^{2}(\mathbb{R})$ to $\left[\operatorname{span}\left\{\phi_{0}\right\}\right]^{\perp} \subset L^{2}(\mathbb{R})$.

${ }^{5}$ Note that $P \phi_{0}=\phi_{0}$ because $\left\langle f_{0}, \phi_{0}\right\rangle=0$ and $P$ is an orthogonal projection to $\left[\operatorname{span}\left\{f_{0}\right\}\right]^{\perp}$.
} 
$\left.\sigma_{e}(A)\right)$. Moreover, this is the only small negative eigenvalue of $A_{\delta}$ for small positive $\sqrt{6}$. Thus, we conclude that if $\left\langle K \phi_{0}, \phi_{0}\right\rangle=\left\langle\mathcal{L}^{-1} \phi_{0}, \phi_{0}\right\rangle<0$, then

$$
\operatorname{dim}\left(\mathcal{H}_{A_{\delta}}^{-}\right)=n(A)+1=n(\mathcal{L}) .
$$

On the other hand, if $\left\langle K \phi_{0}, \phi_{0}\right\rangle>0$, the operator $A_{\delta}=A+\delta K$ is strictly positive on the subspace $\mathcal{H}_{\delta}$ for small positive $\delta$. Therefore, in this case, we have

$$
\operatorname{dim}\left(\mathcal{H}_{A_{\delta}}^{-}\right)=n(A)=n(\mathcal{L}) .
$$

The assertion of the lemma is proven in both the cases.

We are now ready to use Theorem 1 from [13]. Note that although the theorem was proven under the assumption that the essential spectrum of $A$ is bounded away from zero, the shift of $A$ to $A_{\delta}$ satisfying $\sigma_{e}\left(A_{\delta}\right) \geq d_{0} \delta>0$ justifies the technique behind the proof of Theorem 1 in [13] for a small positive number $\delta$. To formulate the theorem, we introduce some notations for the numbers of particular eigenvalues $\gamma$ of the generalized eigenvalue problem (3.7) with the account of their algebraic multiplicities.

- $N_{p}^{-}\left(N_{n}^{-}\right)$is the number of negative eigenvalues $\gamma$ whose (generalized) eigenvectors are associated to the non-negative (non-positive) values of the quadratic form $\langle K \cdot, \cdot\rangle$.

- $N_{p}^{+}\left(N_{n}^{+}\right)$is the number of positive eigenvalues $\gamma$ whose (generalized) eigenvectors are associated to the non-negative (non-positive) values of the quadratic form $\langle K \cdot, \cdot\rangle$.

- $N_{p}^{0}\left(N_{n}^{0}\right)$ is the multiplicity of zero eigenvalue whose (generalized) eigenvectors are associated to the non-negative (non-positive) values of the quadratic form $\langle K \cdot, \cdot\rangle$.

- $N_{c^{+}}\left(N_{c^{-}}\right)$is the number of complex eigenvalues $\gamma$ in the upper (lower) half-plane. Because $A$ and $K$ are real-valued, we have $N_{c^{+}}=N_{c^{-}}$.

We are now ready to reformulate Theorem 1 from [13].

Theorem 2 [13] Under assumptions (H1)-(H4), for a small positive number $\delta$, eigenvalues of the generalized eigenvalue problem (3.13) are counted as follows:

$$
\begin{aligned}
& N_{p}^{-}+N_{n}^{0}+N_{n}^{+}+N_{c^{+}}=\operatorname{dim}\left(\mathcal{H}_{A_{\delta}}^{-}\right), \\
& N_{n}^{-}+N_{n}^{0}+N_{n}^{+}+N_{c^{+}}=\operatorname{dim}\left(\mathcal{H}_{K}^{-}\right) .
\end{aligned}
$$

To apply Theorem 2 to the count of isolated and embedded eigenvalues in the stability problem (1.2), we recall from (3.15) and (3.17) that $\operatorname{dim}\left(\mathcal{H}_{K}^{-}\right)=n(\mathcal{L})$ and $\operatorname{dim}\left(\mathcal{H}_{A_{\delta}}^{-}\right)=n(\mathcal{L})$. At the same time, definition of $N_{n}^{0}$ yields $N_{n}^{0}=n_{0}$, where $n_{0}$ is introduced in Lemma 5. Using these counts, we rewrite equalities (3.19) and (3.20) in the more explicit form:

$$
\begin{aligned}
& N_{p}^{-}+N_{n}^{+}+N_{c^{+}}=n(\mathcal{L})-n_{0}, \\
& N_{n}^{-}+N_{n}^{+}+N_{c^{+}}=n(\mathcal{L})-n_{0} .
\end{aligned}
$$

\footnotetext{
${ }^{6}$ The edge of $\sigma_{e}(A)$ may generate additional eigenvalues by means of edge bifurcations [24]. All these eigenvalues are strictly positive because they detach from the bottom of $\sigma_{e}\left(A_{\delta}\right)$ which is as small as $\mathcal{O}(\delta)$, whereas the distance of these eigenvalues from the bottom of $\sigma_{e}\left(A_{\delta}\right)$ may only change as a superlinear function of $\delta$ as $\delta \rightarrow 0$ [24]. Therefore, all these eigenvalues via edge bifurcations are necessarily positive.
} 
We now need to compute numbers $N_{p}^{-}, N_{n}^{-}, N_{n}^{+}$, and $N_{c^{+}}$for real and complex eigenvalues of the generalized eigenvalue problem (3.7), which are related to real, imaginary, and complex eigenvalues of the spectral problem (1.2). Note that the imaginary eigenvalues of the spectral problem (1.2) may be embedded into the continuous spectrum of the operator $\partial_{x} \mathcal{L}$.

We recall here again the Hamiltonian symmetry, that is, if $\lambda \neq 0$ is a simple eigenvalue of the spectral problem (1.2), then $-\lambda$ is also a simple eigenvalue of the spectral problem (1.2) and both $\lambda$ and $-\lambda$ correspond to the same double eigenvalue $\gamma=-\lambda^{2} \neq 0$ of the generalized eigenvalue problem (3.7).

Lemma 7 Let $\lambda_{j} \in \mathbb{R}_{+}$and $\tilde{\lambda}_{j}=-\lambda_{j} \in \mathbb{R}_{-}$be simple eigenvalues of the spectral problem (1.2) associated with the real-valued eigenvectors $v_{j}$ and $\tilde{v}_{j}$ in $D\left(\partial_{x} \mathcal{L}\right) \cap \dot{H}^{-1}(\mathbb{R})$. Then, we have

$$
\left\langle\mathcal{L} v_{j}^{ \pm}, v_{j}^{ \pm}\right\rangle= \pm 2\left\langle\mathcal{L} \tilde{v}_{j}, v_{j}\right\rangle, \quad\left\langle\mathcal{L} v_{j}^{ \pm}, v_{j}^{\mp}\right\rangle=0,
$$

where $v_{j}^{ \pm}=v_{j} \pm \tilde{v}_{j}$ are linearly independent.

Proof. We recall that the eigenvectors $v_{j}$ and $\tilde{v}_{j}$ for distinct simple eigenvalues $\lambda_{j}$ and $\tilde{\lambda}_{j}$ are linear independent, hence the linear combinations $v_{j}^{+}$and $v_{j}^{-}$are linearly independent. Since $\lambda_{j} \neq 0$ and $v_{j}$ is real-valued, we have

$$
\left\langle\mathcal{L} v_{j}, v_{j}\right\rangle=\frac{1}{\lambda_{j}}\left\langle\mathcal{L} v_{j}, \partial_{x} \mathcal{L} v_{j}\right\rangle=0 .
$$

Similarly, $\left\langle\mathcal{L} \tilde{v}_{j}, \tilde{v}_{j}\right\rangle=0$. The orthogonality relations (3.23) hold by direct computations.

Lemma 8 Let $\lambda_{j} \in i \mathbb{R}_{+}$and $\bar{\lambda}_{j}=-\lambda_{j} \in i \mathbb{R}_{-}$be simple eigenvalues of the spectral problem (1.2) associated with the eigenvectors $v_{j}$ and $\bar{v}_{j}$ in $D\left(\partial_{x} \mathcal{L}\right) \cap \dot{H}^{-1}(\mathbb{R})$. Then, we have

$$
\left\langle\mathcal{L} v_{j}^{ \pm}, v_{j}^{ \pm}\right\rangle=2\left\langle\mathcal{L} v_{j}, v_{j}\right\rangle, \quad\left\langle\mathcal{L} v_{j}^{ \pm}, v_{j}^{\mp}\right\rangle=0,
$$

where $v_{j}^{ \pm}=v_{j} \pm \bar{v}_{j}$ are linearly independent and $\left\langle\mathcal{L} v_{j}, v_{j}\right\rangle$ is real.

Proof. Since operator $\mathcal{L}$ is real-valued, the eigenvector $v_{j}$ of the spectral problem (1.2) with $\operatorname{Im}\left(\lambda_{j}\right) \neq 0$ has both real and imaginary parts. Since $\lambda_{j} \neq 0$, we have

$$
\left\langle\mathcal{L} \bar{v}_{j}, v_{j}\right\rangle=\frac{1}{\lambda_{j}}\left\langle\mathcal{L} \bar{v}_{j}, \partial_{x} \mathcal{L} v_{j}\right\rangle=0 .
$$

Furthermore, since $\mathcal{L}$ is self-adjoint, we have $\left\langle\mathcal{L} v_{j}, v_{j}\right\rangle=\left\langle\mathcal{L} \bar{v}_{j}, \bar{v}_{j}\right\rangle$. The orthogonality equations (3.24) hold by direct computations.

Proof of Theorem 1. By symmetries of the linearized Hamiltonian system, each eigenvalue $\gamma_{j}=-\lambda_{j}^{2}$ of the generalized eigenvalue problem (3.7) has a double multiplicity compared to the eigenvalue $\lambda_{j}$ of the spectral problem (1.2). From two linearly independent eigenvectors $v_{j}^{ \pm} \in$ $D\left(\partial_{x} \mathcal{L}\right) \cap \dot{H}^{-1}(\mathbb{R}) \subset L^{2}(\mathbb{R})$ constructed in Lemmas 7 and 8 , we obtain two linearly independent eigenvectors $w_{j}^{ \pm}=\partial_{x}^{-1} v_{j}^{ \pm} \in \mathcal{H}$ of the generalized eigenvalue problem (3.7) 7 .

\footnotetext{
${ }^{7}$ The same count holds in the case of complex eigenvalues $\lambda_{j}$ with $\operatorname{Re}\left(\lambda_{j}\right) \neq 0$ and $\operatorname{Im}\left(\lambda_{j}\right) \neq 0$. If $v_{j}$ and $\tilde{v}_{j}$ denote linearly independent eigenvectors of the spectral problem (1.2) for complex eigenvalues $\lambda_{j}$ and $-\lambda_{j}$, then we can define two linearly independent eigenvectors $w_{j}=\partial_{x}^{-1} v_{j}$ and $\tilde{w}_{j}=\partial_{x}^{-1} \tilde{v}_{j}$ of the generalized eigenvalue problem (3.7) in $\mathcal{H}$ for the double eigenvalue $\gamma_{j}=-\lambda_{j}^{2}$. In the case of complex eigenvalues, we do not care about the values of the quadratic form associated with the operator $\mathcal{L}$ computed at the eigenvectors.
} 
By the orthogonality condition (3.23), we have $N_{n}^{-}=N_{p}^{-}$for a negative eigenvalue $\gamma_{j}=-\lambda_{j}^{2}$ corresponding to two real eigenvalues $\lambda_{j}$ and $-\lambda_{j}$. Since $N_{n}^{-}+N_{p}^{-}=2 N_{r}$ because of the double multiplicity of eigenvalues $\gamma_{j}$ compared to the multiplicity of eigenvalues $\lambda_{j}$, we obtain $N_{n}^{-}=N_{p}^{-}=$ $N_{r}$. Similarly, for a complex eigenvalue $\gamma_{j}=-\lambda_{j}^{2}$ corresponding to two complex eigenvalues $\lambda_{j}$ and $-\lambda_{j}$, we count $N_{c^{+}}=2 N_{c}$.

By the orthogonality condition (3.24), the double multiplicity of the positive eigenvalue $\gamma_{j}=-\lambda_{j}^{2}$ corresponding to two imaginary eigenvalues $\lambda_{j}$ and $\bar{\lambda}_{j}=-\lambda_{j}$, and the definition of $N_{i}^{-}$, we obtain $N_{n}^{+}=2 N_{i}^{-}$. The count (1.3) follows equivalently from either equality (3.21) or (3.22) 8 .

Remark 2 The count of eigenvalues provided by the equality (3.20) in Theorem 2 is a sufficient tool to prove Theorem 11 since it follows from definitions that $\operatorname{dim}\left(\mathcal{H}_{K}^{-}\right)=n(\mathcal{L}), N_{n}^{0}=n_{0}$, whereas it follows from Lemmas 7 and 8 that $N_{n}^{-}=N_{r}, N_{n}^{+}=2 N_{i}^{-}$, and $N_{c^{+}}=2 N_{c}$. Along this avenue, the count of negative eigenvalues of operators $\mathcal{M}, A$, and $A_{\delta}$ in Lemmas $\mathbf{2}$, 5, and 6 (which is not so easy to prove) is redundant and unnecessary.

\section{Generalization of Theorem 1 for a periodic nonlinear wave}

We shall now take Remark 2 into account for an easy proof of the instability index count for periodic waves in the KdV-type evolution equations. These instability index counts were reported in [7, 17, 21] by means of much longer and different analysis.

We now consider a $2 L$-periodic nonlinear wave $\phi$ in a subspace $\mathcal{X}$ of Hilbert space $L_{\text {per }}^{2}(-L, L)$ equipped with the inner product $\langle\cdot, \cdot \sqrt{9}$ and an induced norm $\|\cdot\|$. The spectral stability of $\phi$ is still determined by the spectral problem (1.2), where $\mathcal{L}:=E^{\prime \prime}(\phi)$ is a self-adjoint real-valued operator with a dense domain $D(\mathcal{L})$ in $L_{\text {per }}^{2}(-L, L)$. We assume that $\mathcal{L}$ has a compact resolvent, so that the spectrum of $\mathcal{L}$ in $L_{\text {per }}^{2}(-L, L)$ is purely discrete. We reinforce assumptions (H2) and (H3) in the slightly modified form:

(H1) The spectrum of $\mathcal{L}$ is purely discrete and includes a finite number $n(\mathcal{L})$ of negative eigenvalues with eigenvectors in $D(\mathcal{L}) \subset L_{\text {per }}^{2}(-L, L)$.

(H2) $\operatorname{Ker}(\mathcal{L})=\operatorname{span}\left\{f_{0}\right\}$ with $f_{0} \in D(\mathcal{L}) \cap D\left(\partial_{x} \mathcal{L} \partial_{x}\right) \cap \dot{H}_{\text {per }}^{-1}(-L, L)$, so that $\phi_{0}=\partial_{x}^{-1} f_{0} \in$ $L_{\mathrm{per}}^{2}(-L, L)$.

In addition, we note that $\operatorname{Ker}\left(\partial_{x}\right)=\operatorname{span}\{1\} \subset L_{\text {per }}^{2}(-L, L)$ and define the matrix $\mathcal{D}$ as follows:

$$
\mathcal{D}=\left[\begin{array}{cc}
\left\langle\mathcal{L}^{-1} \phi_{0}, \phi_{0}\right\rangle & \left\langle\mathcal{L}^{-1} \phi_{0}, 1\right\rangle \\
\left\langle\mathcal{L}^{-1} \phi_{0}, 1\right\rangle & \left\langle\mathcal{L}^{-1} 1,1\right\rangle
\end{array}\right]
$$

Note that matrix $\mathcal{D}$ has finite elements because $\operatorname{span}\left\{1, \phi_{0}\right\} \perp \operatorname{Ker}(\mathcal{L})$ as it follows from the orthogonality conditions $\left\langle f_{0}, \phi_{0}\right\rangle=\left\langle\partial_{x} \phi_{0}, \phi_{0}\right\rangle=0$ and $\left\langle f_{0}, 1\right\rangle=\left\langle\partial_{x} \phi_{0}, 1\right\rangle=0$. We modify now assumption (H4) as follows:

(H3) Matrix $\mathcal{D}$ is invertible.

\footnotetext{
${ }^{8}$ From comparison between (3.19) and (3.20), which is justified by Lemma 4 and the equality $N_{p}^{-}=N_{n}^{-}$, we obtain $\operatorname{dim}\left(\mathcal{H}_{A_{\delta}}^{-}\right)=\operatorname{dim}\left(\mathcal{H}_{K}^{-}\right)=n(\mathcal{L})$, which yields the second independent proof of Lemma 6

${ }^{9}$ Note that we do not change notations for the inner product compared to the case $L^{2}(\mathbb{R})$ but understand that the integration is now performed on $[-L, L]$.
} 
With the previous definitions of $N_{r}, N_{c}$, and $N_{i}^{-}$, the following theorem gives a modification of Theorem 1 for a periodic nonlinear wave.

Theorem 3 Assume (H1)-(H3). Then,

$$
N_{r}+2 N_{c}+2 N_{i}^{-}=n(\mathcal{L})-n(\mathcal{D}),
$$

where $n(\mathcal{D})$ is the number of negative eigenvalues of the matrix $\mathcal{D}$.

Proof. We extend the spectral problem (1.2) to the system of two coupled equations:

$$
\mathcal{M} w=-\lambda v, \quad \mathcal{L} v=\lambda w,
$$

where $\mathcal{M}=-\partial_{x} \mathcal{L} \partial_{x}, v \in D\left(\partial_{x} \mathcal{L}\right) \cap \dot{H}_{\text {per }}^{-1}(-L, L) \subset L_{\text {per }}^{2}(-L, L)$, and $w \in D\left(\partial_{x} \mathcal{L} \partial_{x}\right) \subset L_{\text {per }}^{2}(-L, L)$. The equivalence of simple eigenvalues of the coupled system (4.3) and those of the spectral problem (1.2) is proved similarly to Proposition 1.

By assumptions (H1) and (H2), the zero eigenvalue of $\mathcal{L}$ is isolated and simple. Let $P$ be the orthogonal projection from $L_{\text {per }}^{2}(-L, L)$ to $\left[\operatorname{span}\left\{f_{0}\right\}\right]^{\perp} \subset L_{\text {per }}^{2}(-L, L)$. By a procedure that is similar to (3.8), (3.9), and (3.10), we obtain the generalized eigenvalue problem for a nonzero eigenvalue $\gamma \neq 0$ :

$$
A w=\gamma K w, \quad w \in \mathcal{H}
$$

where $A:=P \mathcal{M} P, K:=P \mathcal{L}^{-1} P, \gamma:=-\lambda^{2}$, and $\mathcal{H}:=D(\mathcal{M}) \cap\left[\operatorname{span}\left\{f_{0}\right\}\right]^{\perp} \subset L_{\text {per }}^{2}(-L, L)$. The equivalence of double eigenvalues of the generalized eigenvalue problem (4.4) and pairs of simple eigenvalues of the coupled system (4.3) is proved similarly to Proposition 3 ,

The spectrum of $\mathcal{M}$ is purely discrete but the zero eigenvalue of $\mathcal{M}$ is now double since $\operatorname{Ker}(\mathcal{M})=$ $\operatorname{span}\left\{1, \phi_{0}\right\}$. Therefore, for a small positive number $\delta$, assumptions of Theorem 2 are satisfied and the equality (3.20) takes the form:

$$
N_{n}^{-}+N_{n}^{0}+N_{n}^{+}+N_{c^{+}}=\operatorname{dim}\left(\mathcal{H}_{K}^{-}\right) .
$$

By construction of $K=P \mathcal{L}^{-1} P$ and $\mathcal{H}=D(\mathcal{M}) \cap\left[\operatorname{span}\left\{f_{0}\right\}\right]^{\perp} \subset L_{\text {per }}^{2}(-L, L)$, we have $\operatorname{dim}\left(\mathcal{H}_{K}^{-}\right)=$ $n(\mathcal{L})$. On the other hand, $N_{n}^{0}$ denotes algebraic multiplicity of zero eigenvalues of the generalized eigenvalue problem (4.4) whose generalized eigenvectors are associated to non-positive values of the quadratic form $\langle K \cdot, \cdot\rangle$. Since $\operatorname{Ker}(\mathcal{M})=\operatorname{span}\left\{1, \phi_{0}\right\}$ and the matrix $\mathcal{D}$ has no zero eigenvalue by assumption (H3), we have $N_{n}^{0}=n(\mathcal{D})$.

From analysis identical to Lemmas 7 and 8, we also obtain $N_{n}^{-}=N_{r}, N_{n}^{+}=2 N_{i}^{-}$, and $N_{c^{+}}=$ $2 N_{c}$, hence the count (4.5) yields the instability index count (4.2) and the theorem is proven.

Remark 3 Equality (3.19) in Theorem 0 can also be used for the correct instability index count but this task would require the count of negative eigenvalues of operators $\mathcal{M}, A$, and $A_{\delta}$ similar to that in Lemmas 2, 5, and 6, which would result in the formula $\operatorname{dim}\left(\mathcal{H}_{A+\delta K}^{-}\right)=n(\mathcal{L})$ for a small positive number 10. For the spectral problem associated with the KdV-type evolution equation, this

\footnotetext{
${ }^{10}$ The identity $\operatorname{dim}\left(\mathcal{H}_{A+\delta K}^{-}\right)=n(\mathcal{L})$ follows from the identity $n(A)=n(P \mathcal{M} P)=n(\mathcal{L})-n(\mathcal{D})$, which should hold despite the fact that the projection operator $P$ is defined by the orthogonal complement of the one-dimensional subspace $\operatorname{Ker}(L)=\operatorname{span}\left\{f_{0}\right\}$. The corresponding argument goes as follows. To study $n(P \mathcal{M})$, we introduce a Lagrange multiplier $\nu$ and set up the self-adjoint spectral problem $\mathcal{M} w=\mu w+\nu f_{0}$ with the orthogonality condition $\left\langle f_{0}, w\right\rangle=0$. Since $\mathcal{M}=-\partial_{x} \mathcal{L} \partial_{x}, f_{0}=\partial_{x} \phi_{0}$, and $\operatorname{Ker}\left(\partial_{x}\right)=\operatorname{span}\{1\}$, we set $w=\partial_{x} g$, integrate in $x$, and obtain the non-self-adjoint spectral problem $\mathcal{L}\left(-\partial_{x}^{2}\right) g=\mu g+\nu \phi_{0}+\chi$ with two Lagrange multipliers $\nu$ and $\chi$, under the constraints $\left\langle\phi_{0}, \partial_{x}^{2} g\right\rangle=0$ and $\left\langle 1, \partial_{x}^{2} g\right\rangle=0$. Smoothing it with a positive parameter $\epsilon$ and setting $g=\left(\epsilon-\partial_{x}^{2}\right)^{-1} h$, we end up with the self-adjoint spectral problem $\mathcal{L} h=\mu\left(\epsilon-\partial_{x}^{2}\right)^{-1} h+\nu \phi_{0}+\chi$ under the constraints $\left\langle\phi_{0}, h\right\rangle=0$ and $\langle 1, h\rangle=0$, which can be studied with the standard technique (Theorem 4.1 in [36]).
} 
equality is redundant because the spectral problem for the coupled system (4.3) is a squared version of the original spectral problem (1.2).

Remark 4 Eigenvectors of the spectral problem (1.2) in $L_{\mathrm{per}}^{2}(-L, L)$ for a nonzero eigenvalue $\lambda$ are orthogonal to $\operatorname{Ker}(\mathcal{M})=\operatorname{span}\left\{1, \phi_{0}\right\}$. Therefore, we can introduce a constrained space

$$
\tilde{\mathcal{H}}:=D(\mathcal{L}) \cap\left[\operatorname{span}\left\{1, \phi_{0}\right\}\right]^{\perp} \subset L_{\text {per }}^{2}(-L, L)
$$

and a projection operator $\tilde{P}: L_{\mathrm{per}}^{2}(-L, L) \rightarrow \tilde{\mathcal{H}}$ to reformulate the spectral problem (1.2) as a linearized Hamiltonian system with an invertible symplectic matrix:

$$
\mathcal{L}_{p} v=\lambda J_{p} v, \quad v \in \tilde{\mathcal{H}}
$$

where $\mathcal{L}_{p}:=\tilde{P} \mathcal{L} \tilde{P}$ and $J_{p}:=\tilde{P} \partial_{x}^{-1} \tilde{P}$. Standard analysis in constrained Hilbert spaces (Theorem 4.1 in [36]) shows that $n\left(\mathcal{L}_{p}\right)=n(\mathcal{L})-n(\mathcal{D})$. Applying now the general instability index count in the linearized Hamiltonian systems with an invertible symplectic operator [23], one can immediately obtain the instability index count formula (4.2). This proof of the instability index count for the nonlinear periodic waves in Hamiltonian systems was introduced by Haragus 85 Kapitula [21].

Let us show how to recover the correct count of eigenvalues for the example of the focusing modified $\mathrm{KdV}$ equation

$$
u_{t}+3 u^{2} u_{x}+u_{x x x}=0 .
$$

Travelling periodic waves in the form $u=\phi(x-c t)$ satisfies the differential equation

$$
\phi^{\prime \prime}=c \phi-\phi^{3},
$$

where the constant of integration is chosen to be zero. Two families of nonlinear periodic waves were considered by Deconinck \& Kapitula [17] in the explicit form:

$$
\begin{aligned}
& \phi(x)=\sqrt{2} k \operatorname{dn}(x, k), \quad c=2-k^{2}, \\
& \phi(x)=\sqrt{2} k \operatorname{cn}(x, k), \quad c=-1+2 k^{2},
\end{aligned}
$$

where dn and cn are Jacobi's elliptic functions and the period $L=4 K(k)$ is given by the complete elliptic integral of the first kind for a fixed $k \in(0,1)$.

Since $\mathcal{L}:=-\partial_{x}^{2}+c-3 \phi^{2}(x)$ with $\mathcal{L} \partial_{x} \phi=0$ and $\mathcal{L} \partial_{c} \phi=-\phi$, assumption (H2) is satisfied with $\phi_{0}=\phi$. In addition, by scaling invariance of the stationary modified KdV equation (4.8), one can obtain that

$$
\left\langle\mathcal{L}^{-1} \phi, \phi\right\rangle=-\frac{1}{2} \frac{d}{d c}\|\phi\|^{2}<0
$$

and

$$
\left\langle\mathcal{L}^{-1} \phi, 1\right\rangle=-\frac{d}{d c} \int_{-L}^{L} \phi(x) d x=0
$$

Let us denote

$$
F(k):=\left\langle\mathcal{L}^{-1} 1,1\right\rangle=\frac{1}{2 L} \int_{-L}^{L} \mathcal{L}^{-1}(1) d x
$$


For the dn-wave, explicit computations in [17] show that $n(\mathcal{L})=1$ and $F(k)>0$ for all $k \in(0,1)$. Therefore, the instability index count (4.2) with $n(\mathcal{L})=n(\mathcal{D})=1$ yields spectral stability of the dn-wave for all $k \in(0,1)$.

For the cn-wave, explicit computations in 17 . show that $n(\mathcal{L})=2$, whereas there is $k_{*} \approx 0.909$ such that $F(k)<0$ for $0<k<k^{*}$ and $F(k)>0$ for $k^{*}<k<1$. Therefore, the instability index count (4.2) yields spectral stability of the cn-wave for $k \in\left(0, k_{*}\right)$ with $n(\mathcal{L})=n(\mathcal{D})=2$ and spectral instability for $k \in\left(k_{*}, 1\right)$ with $n(\mathcal{L})=2, n(\mathcal{D})=1$, and $N_{r}=1$.

\section{Conclusion}

Although the instability index count is now well established both for solitary waves and periodic waves of the $\mathrm{KdV}$-type evolution equations, there are still many directions of further development in the stability theory of nonlinear waves. In particular, Boussinesq equations involve the spectral problem (1.2) associated with a matrix differential operator $\mathcal{L}$ [31. Although this matrix operator can be mapped to a self-adjoint form with a similarity transformation, it becomes difficult to transform both operators $\mathcal{L}$ and $\mathcal{M}$ in the coupled system (3.1) to the self-adjoint form. More direct approaches to the stability of solitary waves in Boussinesq systems can be found in recent works [20, 40. The same complication may also occur in the system of coupled KdV-type equations.

Further extensions of the instability index count involves quadratic operator pencils, as well as general polynomial pencils, where the count of unstable eigenvalues become less precise. Works in this direction can be found in [8, 14, 28].

\section{References}

[1] J. Angulo Pava, Nonlinear Dispersive Equations (Existence and Stability of Solitary and Periodic Travelling Wave Solutions) (AMS, Providence, 2009).

[2] J. Angulo Pava, F.M.A. Natali, "Positivity properties of the Fourier transform and the stability of periodic travelling-wave solutions", SIAM J. Math. Anal. 40 (2008), 1123-1151.

[3] J. Angulo, M. Scialom, and C. Banquet, "The regularized BenjaminOno and BBM equations: well-posedness and nonlinear stability", J. Diff. Eqs. 250 (2011) 4011-4036.

[4] J.L. Bona, P.E. Souganidis, and W.A. Strauss, "Stability and instability of solitary waves of Korteweg-de Vries type", Proc. Roy. Soc. Lond. A 411 (1987), 395-412.

[5] T.J. Bridges and G. Derks, "Linear instability of solitary wave solutions of the Kawahara equation and its generalizations", SIAM J. Math. Anal. 33 (2002), 1356-1378.

[6] T.J. Bridges, G. Derks, and G. Gottwald, "Stability and instability of solitary waves of the fifth-order KdV equation: a numerical framework", Physica D 172 (2002), 190-216.

[7] J.C. Bronski, M.A. Johnson, and T. Kapitula, "An index theorem for the stability of periodic traveling waves of KdV type", Proc. Roy. Soc. Edin. A 141 (2012), 1141-1173.

[8] J.C. Bronski, M.A. Johnson, and T. Kapitula, "An instability index theory for quadratic pencils and applications", arXiv:1207.3764 (2012). 
[9] A.R. Champneys and M.D. Groves, "A global investigation of solitary-wave solutions to a two-parameter model for water waves", J. Fluid Mech. 342 (1997), 199-229.

[10] F. Chardard, F. Dias, and T.J. Bridges, "Computing the Maslov index of solitary waves. I: Hamiltonian systems on a four-dimensional phase space", Physica D 238 (2009), 1841-1867.

[11] M. Chugunova and D. Pelinovsky, "Two-pulse solutions in the fifth-order KdV equation: rigorous theory and numerical approximations", Discr. Cont. Dyn. Syst. B 8 (2007), 773800 .

[12] M. Chugunova and D Pelinovsky, "Count of unstable eigenvalues in the generalized eigenvalue problem", arXiv:math/0602386 (2006).

[13] M. Chugunova and D Pelinovsky, "Count of unstable eigenvalues in the generalized eigenvalue problem", J. Math. Phys. 51 (2010), 052901 (19 pages).

[14] M. Chugunova and D. Pelinovsky, "On quadratic eigenvalue problems arising in stability of discrete vortices", Lin. Alg. Appl. 431 (2009), 962-973.

[15] W. Craig and M.D. Groves, "Hamiltonian long-wave approximations to the water-wave problem", Wave Motion 19 (1994), 367-389.

[16] S. Cuccagna, D. Pelinovsky, and V. Vougalter, "Spectra of positive and negative energies in the linearized NLS problem", Comm. Pure Appl. Math. 58 (2005), 1-29.

[17] B. Deconinck and T. Kapitula, "On the spectral and orbital stability of spatially periodic stationary solutions of generalized Korteweg-de Vries equations", preprint online at

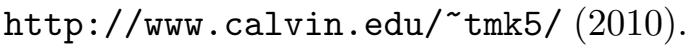

[18] F. Dias and E.A. Kuznetsov, "Nonlinear stability of solitons in the fifth-order KdV equation", Phys. Lett. A 263 (1999), 98-104.

[19] M. Grillakis, J. Shatah, and W. Strauss, "Stability theory of solitary waves in the presence of symmetry", J. Funct. Anal. 74 (1987), 160-197; J. Funct. Anal. 94 (1990), 308-348.

[20] S. Hakkaev, M. Stanislavova, and A. Stefanov, "Spectral stability for subsonic traveling pulses of the Boussinesq 'ABC' system", SIAM J. Appl. Dyn. Syst., to be published (2013).

[21] M. Haragus and T. Kapitula, "On the spectra of periodic waves for infinite-dimensional Hamiltonian systems", Physica D 237 (2008), 2649-2671.

[22] A.T. Illichev and A.Y. Semenov, "Stability of solitary waves in dispersive media described by a fifth-order evolution equation", Theor. Comp. Fluid Dyn. 3 (1992), 307-326.

[23] T. Kapitula, P. Kevrekidis, and B. Sandstede, "Counting eigenvalues via the Krein signature in infinite-dimensional Hamiltonian systems", Physica D 195 (2004), 263-282; Addendum: Physica D 201 (2005), 199-201.

[24] T. Kapitula and B. Sandstede, "Edge bifurcations for near integrable systems via Evans function techniques", SIAM J. Math. Anal. 33 (2002), 1117-1143.

[25] T. Kapitula and A. Stefanov, "A Hamiltonian-Krein (instability) index theory for KdV-like eigenvalue problems", arXiv: 1210.6005 (2012). 
[26] S. Kichenassamy, "Existence of solitary waves for water-wave models", Nonlinearity 10 (1997), 133-151.

[27] Y. Kodama and D. Pelinovsky, "Spectral stability and time evolution of $N$ solitons in KdV hierarchy", J. Phys. A: Math. Gen. 38 (2005), 6129-6140.

[28] R. Kollar, "Homotopy method for nonlinear eigenvalue pencils with applications", SIAM J. Math. Anal. 43 (2011), 612-633.

[29] R. Kollar and P. Miller, "Graphical Krein signature theory and Evans-Krein functions", arXiv: 1209.3185 (2012).

[30] S.P. Levandovsky, "A stability analysis for fifth-order water-wave models", Physica D 125 (1999), 222-240.

[31] Z. Lin, "Instability of nonlinear dispersive solitary waves", J. Funct. Anal. 255 (2008), 11911224.

[32] J.H. Maddocks and R.L. Sachs, "On the stability of KdV multi-solitons", Comm. Pure Appl. Math. XLVI (1993), 867-901.

[33] R.L. Pego and M.I. Weinstein, "Eigenvalues, and instabilities of solitary waves", Philos. Trans. Roy. Soc. London A 340 (1992), 47-94.

[34] R.L. Pego and M.I. Weinstein, "Asymptotic stability of solitary waves", Comm. Math. Phys. 164 (1994), 305-349.

[35] D.E. Pelinovsky, "Inertia law for spectral stability of solitary waves in coupled nonlinear Schrödinger equations", Proc. Roy. Soc. Lond. A, 461 (2005), 783-812.

[36] D.E. Pelinovsky, Localization in periodic potentials: from Schrödinger operators to the GrossPitaevskii equation (Cambridge University Press, Cambridge, 2011).

[37] D.E. Pelinovsky and P.G. Kevrekidis "Dark solitons in external potentials", Z. angew. Math. Phys. 59 (2008), 559-599.

[38] L.S. Pontryagin, "Hermitian operators in spaces with indefinite metric", Izv. Akad. Nauk SSSR Ser. Mat. 8 (1944), 243-280.

[39] P.E. Souganidis and W.A. Strauss, "Instability of a class of dispersive solitary waves", Proc. Roy. Soc. Edin. A 114 (1990), 195-212.

[40] M. Stanislavova and A. Stefanov, "Linear stability analysis for travelling waves of second order in time PDEs", Nonlinearity 25 (2012), 2625-2654.

[41] M.I. Weinstein, "Existence and dynamic stability of solitary wave solutions of equations arising in long wave propagation", Comm. PDEs 12 (1987), 1133-1173. 